Pacific

Journal of

Mathematics

THE MINIMAL VOLUME ORIENTABLE HYPERBOLIC 3-MANIFOLD WITH 4 CUSPS

KEN'ICHI YOSHIDA 


\title{
THE MINIMAL VOLUME ORIENTABLE HYPERBOLIC 3-MANIFOLD WITH 4 CUSPS
}

\author{
KEN'ICHI YOSHIDA
}

\begin{abstract}
We prove that the $8_{2}^{4}$ link complement is the minimal volume orientable hyperbolic manifold with 4 cusps. Its volume is twice the volume $V_{8}$ of the ideal regular octahedron; that is, 7.32 $\ldots=2 V_{8}$. The proof relies on Agol's argument used to determine the minimal volume hyperbolic 3-manifolds with 2 cusps. We also need to estimate the volume of a hyperbolic 3-manifold with totally geodesic boundary which contains an essential surface with nonseparating boundary.
\end{abstract}

\section{Introduction}

The volumes of hyperbolic 3-manifolds are known to be topological invariants. The structure of the set of the volumes of hyperbolic 3-manifolds is known.

Theorem 1.1 (Jørgensen and Thurston's; see [Benedetti and Petronio 1992, Corollaries E.7.1 and E.7.5]). The set of the volumes of orientable hyperbolic 3-manifolds is a well-ordered set of the type $\omega^{\omega}$ with respect to the order of $\mathbb{R}$. The volume of an orientable hyperbolic 3-manifold with $n$ cusps corresponds to an $n$-fold limit ordinal.

This theorem gives rise to the problem of determining the minimal volume orientable hyperbolic 3-manifolds with $n$ cusps. The answers are known in the cases where $0 \leq n \leq 2$.

- In the case where $n=0$ (closed manifold),

Gabai, Meyerhoff and Milley [2009] showed that the Weeks manifold has the minimal volume. Its volume is $0.94 \ldots$.

- In the case where $n=1$,

Cao and Meyerhoff [2001] showed that the figure-eight knot complement and the manifold obtained by the $(5,1)$-Dehn surgery from the Whitehead link

This research is supported by Global COE Program "New Development in Mathematics" from the Ministry of Education, Culture, Sports, Science and Technology (MEXT) of Japan.

MSC2010: 57M27.

Keywords: hyperbolic 3-manifold, essential surface, geodesic boundary. 

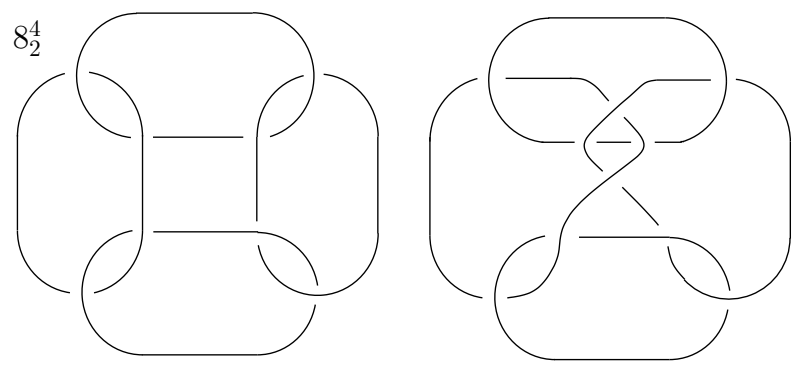

Figure 1. The $8_{2}^{4}$ link and a link whose complement is homeomorphic to that of the $8_{2}^{4}$ link.

complement have the minimal volume. Their volume is $2.02 \ldots=2 V_{3}$, where $V_{3}$ is the volume of the ideal regular tetrahedron.

- In the case where $n=2$,

Agol [2010] showed that the (-2,3,8)-pretzel link complement and the Whitehead link complement have the minimal volume. Their volume is $3.66 \ldots=4 \sum_{k=0}^{\infty}(-1)^{k} /(2 k+1)^{2}=V_{8}$, where $V_{8}$ is the volume of the ideal regular octahedron.

In the case where $n \geq 3$, Adams [1988] showed that the volume of an $n$-cusped hyperbolic 3-manifold is not less than $n V_{3}$. Agol [2010] conjectured the following:

- In the case where $3 \leq n \leq 10$,

the minimally twisted hyperbolic chain link complement has the minimal volume.

- In the case where $n \geq 11$,

the $(n-1)$-fold covering of Whitehead link complement has the minimal volume.

In this paper, we prove this conjecture in the case where $n=4$.

Theorem 1.2. The minimal volume orientable hyperbolic 3-manifold with 4 cusps is homeomorphic to the $8_{2}^{4}$ link complement. Its volume is $7.32 \ldots=2 V_{8}$.

We remark that this link is not the unique one to determine the complement. For example, the complement of the link on the right of Figure 1 is homeomorphic to the $8_{2}^{4}$ link complement.

We will prove Theorem 1.2 in Sections 4 and 5. The proof owes much to Agol [2010].

\section{Review of Agol's argument}

In this section, we set up some notation and review the argument used by Agol [2010] to determine the minimal volume of 2-cusped hyperbolic 3-manifolds. We 
treat compact smooth 3-manifolds with boundary and corners. We only consider surfaces in a compact 3-manifold which are properly embedded or contained in the boundary. Let $I=[0,1]$.

Let $M$ be a 3-manifold with boundary. For a properly embedded surface $X \subset M$, let $M \| X$ denote the path-metric closure of $M-X$. We will say that $X$ is essential if $X$ is incompressible and $\partial$-incompressible and has no component parallel to the boundary. Essential surfaces are not assumed to be connected.

A finite volume orientable hyperbolic 3-manifold can be the interior of a compact 3-manifold with the boundary which consists of tori. Its boundary component is called a cusp. When we say a hyperbolic manifold in what follows, it often means this compact manifold. We also consider hyperbolic manifolds with totally geodesic boundary. In this case there may be annular cusps which adjoin the totally geodesic boundary. The double of a hyperbolic manifold $M$ with totally geodesic boundary is the manifold obtained from two copies of $M$ by gluing along the totally geodesic boundary. Then two annular cusps form one torus cusp in its double.

We introduce the notion of pared manifolds. It was defined by Thurston to characterize a topological property of geometrically finite hyperbolic manifolds.

Definition 2.1 [Thurston 1986, Section 7; Morgan 1984, Definition 4.8]. A pared manifold is a pair $(M, P)$ such that

- $M$ is a compact orientable irreducible 3-manifold,

- $P \subset \partial M$ is a union of annuli and tori which are incompressible in $M$,

- every abelian, noncyclic subgroup of $\pi_{1}(M)$ is peripheral with respect to $P$ (that is, conjugate to a subgroup of the fundamental group of a component of $P)$, and

- every map $\phi:\left(S^{1} \times I, S^{1} \times \partial I\right) \rightarrow(M, P)$ which induces injective maps on the fundamental groups deforms, as maps of pairs, into $P$.

We call $P$ the parabolic locus of the pared manifold $(M, P)$, and an annular component of $P$ is called a pared annulus. We denote the surface $\partial M-\operatorname{int}(P)$ by $\partial_{0} M$.

Let $(M, P)$ be a pared manifold. If every map $\psi:\left(S^{1} \times I, S^{1} \times \partial I\right) \rightarrow\left(M, \partial_{0} M\right)$ that induces injective maps on the fundamental groups deforms either into $\partial_{0} M$ or into $P$, then we call $(M, P)$ acylindrical.

Since a finite volume orientable hyperbolic 3-manifold is atoroidal, it is a pared manifold by letting its parabolic locus be the cusp tori. Conversely:

Theorem 2.2. Let $(M, P)$ be an acylindrical Haken pared manifold, and assume that $\partial_{0} M$ is incompressible. We assume that $M$ is not a 3-ball, a $T^{2} \times I$ or a solid torus. Then $M-P$ admits a finite volume hyperbolic structure with totally geodesic boundary $\partial_{0} M$. This hyperbolic structure is unique up to isometry. 
Since the double $D M$ of an acylindrical pared manifold $(M, P)$ is atoroidal, $D M$ admits a finite volume hyperbolic structure, where $D M$ is obtained from two copies of $M$ by gluing along $\partial_{0} M$. Then the diffeomorphism swapping the two copies of $M$ can be taken to be an isometry. The fixed point set $\partial_{0} M$ is totally geodesic [Leininger 2006, Lemma 2.6].

When a hyperbolic manifold is cut along an essential surface, the obtained manifold is a pared manifold.

Lemma 2.3 [Agol 2010, Lemma 3.2]. Let $M$ be a finite volume orientable hyperbolic 3-manifold, and $\partial M$ be the parabolic locus $P$ of $M$. Let $X \subset M$ be an essential surface. Then $(M \rrbracket X, P \Downarrow \partial X)$ is a pared manifold.

Theorem 2.4 (JSJ decomposition for a pared manifold; see [Jaco and Shalen 1979; Johannson 1979; Morgan 1984, Section 11]). Let (M, P) be a pared manifold such that $\partial_{0} M$ is incompressible. There is a canonical set of essential annuli $(A, \partial A) \subset$ $\left(M, \partial_{0} M\right)$ called the characteristic annuli. It is characterized up to isotopy by the property that they are the maximal collection of nonparallel essential annuli such that every other essential annulus $(B, \partial B) \subset\left(M, \partial_{0} M\right)$ may be relatively isotoped to an annulus $\left(B^{\prime}, \partial B^{\prime}\right) \subset\left(M, \partial_{0} M\right)$ so that $B^{\prime} \cap A=\varnothing$. Then each complementary component $\left(L, \partial_{0} L\right) \subset\left(M \Downarrow A, \partial_{0} M \backslash \partial A\right)$ is one of the following types:

(1) $\left(T^{2} \times I,\left(T^{2} \times I\right) \cap \partial_{0} M\right)$, where one of the boundary components $T^{2} \times \partial I$ is a torus component of $P$.

(2) $\left(S^{1} \times D^{2},\left(S^{1} \times D^{2}\right) \cap \partial_{0} M\right)$, which is a solid torus with annuli in the boundary.

(3) (I-bundle, $\partial$ I-subbundle), which is an I-bundle over a surface whose Euler characteristic is negative, and the I-bundle over the boundary is contained in $A \cup P$.

(4) $\left(L, L \cap \partial_{0} M\right)$, where $L$ has no essential annuli whose boundary is contained in $L \cap \partial_{0} M$.

A neighborhood of a torus component of $P$ is either of type 1 or of type 4 . One of the boundary components $T^{2} \times \partial I$ of type 1 is a torus component of $P$, and the intersection of the other boundary component and $\partial_{0} M$ is a union of essential annuli in the torus. The intersection $\left(S^{1} \times D^{2}\right) \cap \partial_{0} M$ in a component of type 2 is a union of essential annuli in $\partial\left(S^{1} \times D^{2}\right)$. The union of components of type 3 is called the window. A component of type 4 is the acylindrical pared manifold $\left(L, L-\partial_{0} M\right)$. The union of the components of type 4 is called the guts and denoted by $\operatorname{Guts}(M, P)$. A torus boundary component of the guts is a torus component of $P$.

The definition of guts in [Agol 2010] is a bit different from ours. There, the guts are defined to be the union of types 1, 2 and 4. The definition in [Agol et al. 2007] is same as ours, and it is appropriate for our purpose. 
Let $M$ be a finite volume orientable hyperbolic manifold. For an essential surface $X \subset M,(M \Downarrow X, P \Downarrow \partial X)$ is a pared manifold by Lemma 2.3. Therefore, we can define $\operatorname{Guts}(X)=\operatorname{Guts}(M \Downarrow X, P \Downarrow \partial X)$. Then the components of $\operatorname{Guts}(X)$ admit hyperbolic structures with geodesic boundary by Theorem 2.2. Hence the volume vol Guts $(X)$ is defined. This volume is not greater than the volume of $M$.

Theorem 2.5 [Agol et al. 2007, Theorem 9.1]. Let $M$ be a finite volume orientable hyperbolic manifold, and $X \subset M$ be an essential surface. Then

$$
\operatorname{vol} M \geq \operatorname{vol} \operatorname{Guts}(X) \geq \frac{V_{8}}{2}|\chi(\partial \operatorname{Guts}(X))| .
$$

Moreover, the refinement by Calegari, Freedman and Walker (in [Calegari et al. 2010, Theorem 5.5]) implies that $M$ is obtained from ideal regular octahedra by gluing along the faces when the equality holds.

The estimate of vol Guts $(X)$ from below in Theorem 2.5 follows from the following theorem.

Theorem 2.6 [Miyamoto 1994, Theorem 5.2]. Let $M$ be a hyperbolic manifold with totally geodesic boundary. Then vol $M \geq V_{8} / 2|\chi(\partial M)|$. Moreover, $M$ is obtained from ideal regular octahedra by gluing along their faces when the equality holds.

Lemma 2.7. Let $M$ be a finite volume orientable hyperbolic 3-manifold, and $X \subset M$ be a nonempty essential surface. Then each component of $\operatorname{Guts}(X)$ has negative Euler characteristic.

Proof. Since the Euler characteristic of every closed 3-manifold is 0 , we have $\chi(\operatorname{Guts}(X))=\frac{1}{2} \chi(\partial \operatorname{Guts}(X))$. Assume that there is a component $L$ of $\operatorname{Guts}(X)$ such that $\chi(L) \geq 0$. Since no component of $\partial \operatorname{Guts}(X)$ is a sphere, $\chi(L)=0$ and $\partial L$ consists of tori. Since $M$ is atoroidal, $\partial L \subset \partial M$. This implies $L=M$ by connectedness of $M$. This contradicts the fact that $X$ is not empty.

This lemma implies that $\chi(\partial \operatorname{Guts}(X)) \leq-4$ if $\operatorname{Guts}(X)$ is not connected.

We will use annular compressions to obtain a surface whose guts are not empty.

Definition 2.8. Let $(X, \partial X) \subset(M, \partial M)$ be an essential surface in a 3-manifold. A compressing annulus is an embedding $i:\left(S^{1} \times I, S^{1} \times\{0\}, S^{1} \times\{1\}\right) \hookrightarrow(M, X, \partial M)$ such that

- $i_{*}$ induces injective maps on $\pi_{1}$,

- $i\left(S^{1} \times I\right) \cap X=i\left(S^{1} \times\{0\}\right)$, and

- $i\left(S^{1} \times\{0\}\right)$ is not isotopic in $X$ to $\partial X$. 

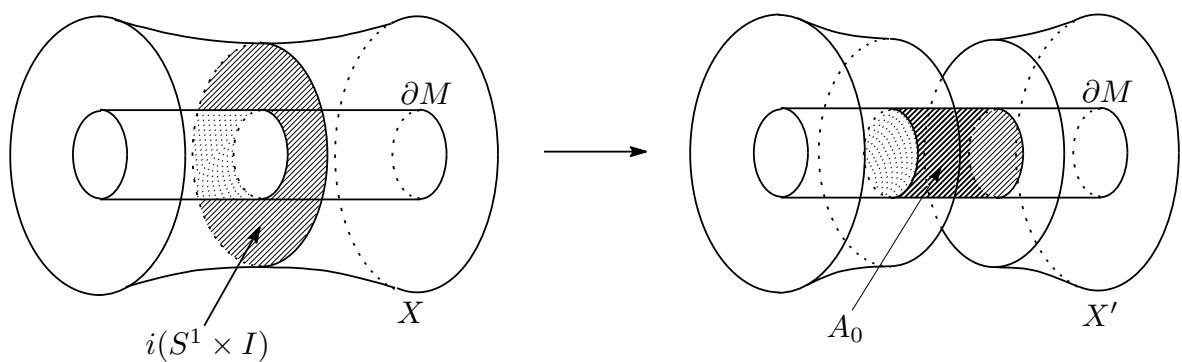

Figure 2. An annular compression.

An annular compression of $(X, \partial X) \subset(M, \partial M)$ is the surgery along a compressing annulus $i\left(S^{1} \times I\right)$. Let $U$ be a regular neighborhood of $i\left(S^{1} \times I\right)$ in $M \| X$, and put $\partial_{0} U=\partial U \cap(X \cup \partial M)$ and $\partial_{1} U=\overline{\partial U-(X \cup \partial M)}$. Then the surface $X^{\prime}=\left(X-\partial_{0} U\right) \cup \partial_{1} U$ is called the annular compression of $X$. If $X$ is essential, $X^{\prime}$ is also essential. We will say that $A_{0}=\partial U \cap \partial M$ is the annulus in the boundary created by the annular compression (Figure 2). This annulus is not contained in the window of $M \| X^{\prime}$.

Lemma 2.9 [Agol 2010, Lemma 3.3]. Let $M$ be a finite volume orientable hyperbolic manifold. Let $X \subset M$ be an essential surface. If $X$ has a compressing annulus, let $X^{\prime}$ be the annular compression of $X$. Then the annulus in the boundary created by this annular compression is not contained in the window of $M \rrbracket X^{\prime}$.

The following lemma is used in the proof of [Agol 2010, Theorem 3.4]. Lemmas 2.9 and 2.10 imply that a torus or an annulus in the boundary is contained in the boundary of the gut regions after we perform annular compressions as many times as possible.

Lemma 2.10. Let $M$ and $X$ be as above. We assume that a $T^{2} \times I$ component or an $S^{1} \times D^{2}$ component intersects a component $T$ of $\partial M$. Then we can perform an annular compression for $X$ toward $T$.

The following theorem is a result in [Culler and Shalen 1984, Theorem 3]. We will use it to find an essential surface to start the proof of Theorem 4.3.

Theorem 2.11. Let $M$ be a finite volume orientable hyperbolic manifold with $n$ cusps, and let $\partial M=T_{1} \cup \cdots \cup T_{n}$, where $T_{i}$ is a torus for $1 \leq i \leq n$. Let $k$ be an integer such that $1 \leq k \leq n$. Then there is an essential surface $X \subset M$ such that $\partial X \cap T_{i} \neq \varnothing$ for $1 \leq i \leq k$ and $\partial X \cap\left(T_{k+1} \cup \cdots \cup T_{n}\right)=\varnothing$.

\section{Essential surfaces in 3-manifold with boundary}

In this section we find an essential surface in a hyperbolic 3-manifold with geodesic boundary. Using this we will estimate the volume of a hyperbolic 3-manifold 
with geodesic boundary with at least 4 cusps. Essential surfaces are found by a homological argument for 3-manifolds, and it is not necessary to assume the hyperbolic structure.

Lemma 3.1 [Hatcher 2007, Lemma 3.5]. Let M be a compact orientable 3-manifold. Then the rank of the boundary homomorphism $\partial_{*}: H_{2}(M, \partial M ; \mathbb{Q}) \rightarrow H_{1}(\partial M ; \mathbb{Q})$ is half of the dimension of $H_{1}(\partial M ; \mathbb{Q})$.

Lemma 3.2. Let $L$ be an orientable hyperbolic 3-manifold with geodesic boundary $S$, with $k$ annular cusps $A_{1}, \ldots, A_{k}$ and with $n-k$ torus cusps $T_{k+1}, \ldots, T_{n}$, where $1 \leq k \leq 3$ and $n \geq 4$. Assume that $\chi(S)=-2$. Then there is an essential surface $Y \subset L$ such that $Y \cap S=\varnothing$ and $[\partial Y] \neq 0 \in H_{1}(\partial L ; \mathbb{Z})$.

Proof. The union $S^{\prime}=S \cup A_{1} \cup \cdots \cup A_{k}$ is a closed surface of genus 2. We note that there are only two types of essential closed curves in $S^{\prime}$; one separates $S^{\prime}$ and the other does not. There are no pairs of disjoint separating curves in $S^{\prime}$.

We can take $k-1$ annuli of $\left\{A_{1}, \ldots, A_{k}\right\}$ such that the complement of them is connected. The image of $\partial_{*}: H_{2}(L, \partial L ; \mathbb{Q}) \rightarrow H_{1}(\partial L ; \mathbb{Q})$ is a subspace of $H_{1}(\partial L ; \mathbb{Q}) \cong \mathbb{Q}^{2(n-k)+4}$ of dimension $n-k+2$, by Lemma 3.1. We consider the subspace $V$ of $H_{1}(\partial L ; \mathbb{Q})$ spanned by all the elements represented by curves in $A_{1}, \ldots, A_{k-1}, T_{k+1}, \ldots, T_{n}$. Since the dimension of $V$ is $2(n-k)+(k-1)$, $V$ intersects $\operatorname{Im}\left(\partial_{*}\right)$ in a nontrivial subspace of $H_{1}(\partial L ; \mathbb{Q})$. Hence there exists a nonzero element $z$ in $H_{2}(L, \partial L ; \mathbb{Q})$ such that $\partial_{*} z \neq 0$ and $z$ belongs to $V$. By taking a multiple of $z$, there exists a nonzero element $z^{\prime}$ in $H_{2}(L, \partial L ; \mathbb{Z})$ such that $\partial_{*} z^{\prime} \neq 0$ and $\partial_{*} z^{\prime}$ is represented by a union of closed curves in $A_{1}, \ldots, A_{k-1}, T_{k+1}, \ldots, T_{n}$. We can find an essential surface $Y$ representing $z^{\prime}$ such that

$$
\partial Y \subset A_{1} \cup \cdots \cup A_{k-1} \cup T_{k+1} \cup \cdots \cup T_{n} .
$$

\section{Estimate of volume}

Now we are going to estimate the volume of a hyperbolic manifold with geodesic boundary. Lemma 3.2 and Theorem 4.1 imply that the volume of an orientable hyperbolic 3-manifold with 4 cusps and with geodesic boundary is not less than $2 V_{8}$.

Theorem 4.1. Let $L$ be an orientable hyperbolic 3-manifold with geodesic boundary $S$. Suppose that there is an essential surface $Y \subset L$ such that $Y \cap S=\varnothing$ and $[\partial Y] \neq 0 \in H_{1}(\partial L ; \mathbb{Z})$. Then there is an essential surface $Y^{\prime}$ such that $\chi\left(\partial \operatorname{Guts}\left(L \rrbracket Y^{\prime}\right)\right) \leq-4$ and vol $L \geq 2 V_{8}$.

If $\chi(S) \leq-4$, then vol $L \geq 2 V_{8}$ by Theorem 2.6. Hence we may assume that $\chi(S)=-2$. Let $S^{\prime}$ denote the surface which is the union of $S$ and the annular cusps of $L$. $\partial L$ consists of $S^{\prime}$ and the torus cusps of $L$.

We will find an essential surface $Y^{\prime}$ such that $\chi\left(\partial \operatorname{Guts}\left(L \rrbracket Y^{\prime}\right)\right) \leq-4$. Then $\chi\left(\partial \operatorname{Guts}\left(D L \|\left(D Y^{\prime} \cup S\right)\right) \leq-8\right.$, where $D L$ is the double of $L$ (the hyperbolic 
manifold obtained from two copies of $L$ by gluing along the geodesic boundary $S$ ) and $D Y^{\prime}$ is the union of two copies of $Y^{\prime}$ in $D L$. Then Theorem 2.5 implies vol $D L \geq 4 V_{8}$, and so vol $L \geq 2 V_{8}$.

We will find a gut component intersecting $S$. For this we need to know how a window component intersects $S$.

Lemma 4.2. Let $L, S$ and $Y$ be as above. Assume that $S$ intersects a component $\left(J, \partial_{0} J\right)$ of the window of $L \rrbracket Y$. Then $\left(J, \partial_{0} J\right)$ is a product I-bundle and intersects $S$ only on one component of the $\partial I$-bundle.

Proof. Suppose that the base space of $J$ is nonorientable. Since $\partial_{0} J$ is connected, $\partial_{0} J \subset S$. We take a simple closed curve $\alpha$ in $J$ such that $\alpha$ is projected to an orientation-reversing loop in the base space of $J$. There is a simple closed curve $\beta$ in $\partial_{0} J$ such that $[\beta]=[\alpha]^{2} \in \pi_{1}(D L) \subset \operatorname{Isom}^{+}\left(\mathbb{M}^{3}\right)$. If $\beta$ is homotopic to the boundary of $\partial_{0} J$, the base space of $J$ is a Möbius band. It contradicts the definition of the window. Hence $[\beta] \in \pi_{1}(S) \subset \operatorname{Isom}^{+}\left(\mathbb{H}^{2}\right)$ is hyperbolic element. The simple closed curve $\beta$ is homotopic to a simple closed geodesic $\beta^{\prime}$ in $S$ [Ratcliffe 2006, Theorem 9.6.5]. But the fact that $\left[\beta^{\prime}\right]=[\alpha]^{2}$ contradicts the fact that an element represented by a simple closed geodesic in a hyperbolic manifold has no roots [Ratcliffe 2006, Theorem 9.6.2]. Therefore no twisted $I$-bundle intersects $S$.

Suppose that the base space of $J$ is orientable and both components $Q_{0}$ and $Q_{1}$ of $\partial_{0} J$ are contained in $S$. Since $\chi\left(Q_{0}\right)=\chi\left(Q_{1}\right)<0$, there are (not necessarily simple) closed curves $\gamma_{i} \subset Q_{i}(i=0,1)$ such that $\gamma_{i}$ is not homotopic to the boundary of $Q_{i}$ and $\gamma_{0}$ and $\gamma_{1}$ are homotopic in $L$. Let $\gamma_{i}^{\prime}$ be the closed geodesic in $Q_{i}$ homotopic to $\gamma_{i}$. Since $L$ is totally geodesic, the two closed geodesics $\gamma_{0}^{\prime}$ and $\gamma_{1}^{\prime}$ are homotopic in $L$. It contradicts the uniqueness of the closed geodesic in a homotopy class. Therefore a product $I$-bundle intersects $S$ on at most one side of the $\partial I$-bundle.

Proof of Theorem 4.1. Let $Y_{0}$ be an essential surface in $L$ such that $Y_{0} \cap S=\varnothing$ and $\left[\partial Y_{0}\right] \neq 0 \in H_{1}(\partial L ; \mathbb{Z})$. Moreover let $\left|\chi\left(Y_{0}\right)\right|$ be minimal among the surfaces satisfying these conditions. Since $L$ has no essential sphere, disk, torus or annulus, $\chi\left(Y_{0}\right)<0$. Let $p: L \rrbracket Y_{0} \rightarrow L$ be the natural projection.

(i) First we consider the case where $S$ intersects a component $\left(J, \partial_{0} J\right)$ of the window of $L \rrbracket Y_{0}$. Then $\chi(J)$ is equal to -1 or -2 . We will show that $\chi(J)=-1$. Assume that $\chi(J)=-2$. $S \cap p(J)$ is a 2-punctured torus or a 4-punctured sphere. (If it is a closed surface, $Y_{0} \cap p(J)$ is a component of $Y_{0}$ which is parallel to $S^{\prime}$. It contradicts that $Y_{0}$ is essential.) Let $Y_{0}^{\prime}$ be the surface which is the union of annuli (Figure 3) and $Y_{0}-\left(Y_{0} \cap p(J)\right)$. If there is an annulus in $L-Y_{0}$ whose boundary is two components of the frontier of $Y_{0}-\left(Y_{0} \cap p(J)\right)$, we glue $Y_{0}-\left(Y_{0} \cap p(J)\right)$ and this annulus (the upper of Figure 3). Since $Y_{0} \cap p(J)$ is connected, the orientation matches. Otherwise, there is an annular cusp which is homotopic to the frontier of 


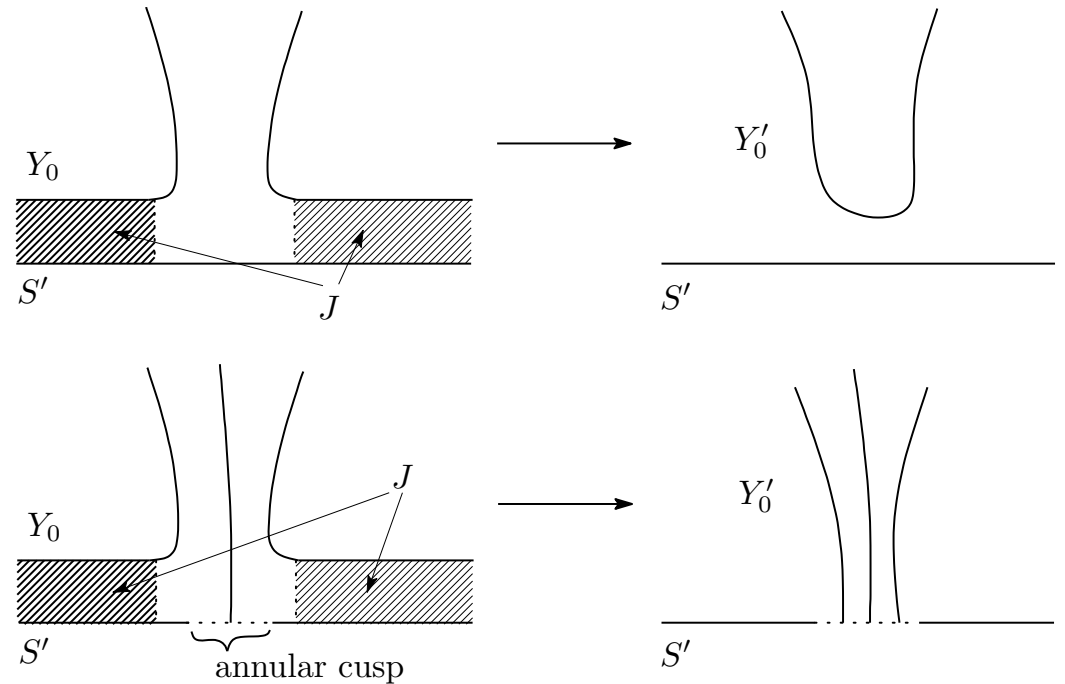

Figure 3. Constructions in the case where $S$ intersects a component of the window whose Euler characteristic is -2 .

$Y_{0}-\left(Y_{0} \cap p(J)\right)$. Then we can glue $Y_{0}-\left(Y_{0} \cap p(J)\right)$ and the two annuli, where one of the boundary components of each annulus is contained in this annular cusp (the lower of Figure 3). Then $\left[Y_{0}^{\prime}, \partial Y_{0}^{\prime}\right]=\left[Y_{0}, \partial Y_{0}\right] \in H_{2}(L, \partial L ; \mathbb{Z})$. We obtain an essential surface from $Y_{0}^{\prime}$ by compressing if necessary. Then $\left|\chi\left(Y_{0}^{\prime}\right)\right|<\left|\chi\left(Y_{0}\right)\right|$, contradicting the choice of $Y_{0}$. Therefore $\chi(J)=-1$.

We will find an essential surface $Y_{1}$ such that $S$ intersects only one component of the window of $L \rrbracket Y_{1}$. If $S$ intersects only one component of the window of $L \rrbracket Y_{0}$ already, put $Y_{1}=Y_{0}$. Suppose that $S$ intersects two components $\left(J, \partial_{0} J\right)$ and $\left(J^{\prime}, \partial_{0} J^{\prime}\right)$ of the window of $L \rrbracket Y_{0}$. Let $Y_{0}^{\prime}$ be the surface which is the union of $Y_{0}-\left(Y_{0} \cap p(J)\right)$ and a surface in $p\left(J^{\prime}\right)$ (Figure 4). Then

$$
\left[Y_{0}^{\prime}, \partial Y_{0}^{\prime}\right]=\left[Y_{0}, \partial Y_{0}\right] \in H_{2}(L, \partial L ; \mathbb{Z}) .
$$

Note that since the orientation may not match, we cannot construct a surface as in Figure 3. If $Y_{0}^{\prime}$ is not essential, we obtain an essential surface simpler than $Y_{0}$ by compressing $Y_{0}^{\prime}$. Since this $Y_{0}^{\prime}$ is essential.

Suppose that $S$ intersects two components of the window of $L \rrbracket Y_{0}^{\prime}$ again. Then one of these two components is contained in $p\left(J^{\prime}\right)$. We can perform the above construction again and remove a part of $Y_{0}^{\prime}$ which is contained in the boundary of the window. Since no $I$-bundle can intersect $S$ essentially along both components of the boundary by Lemma 4.2, the part of the obtained surface in $p\left(J^{\prime}\right)$ is not contained in the boundary of the component of the window which intersects $S$ and 


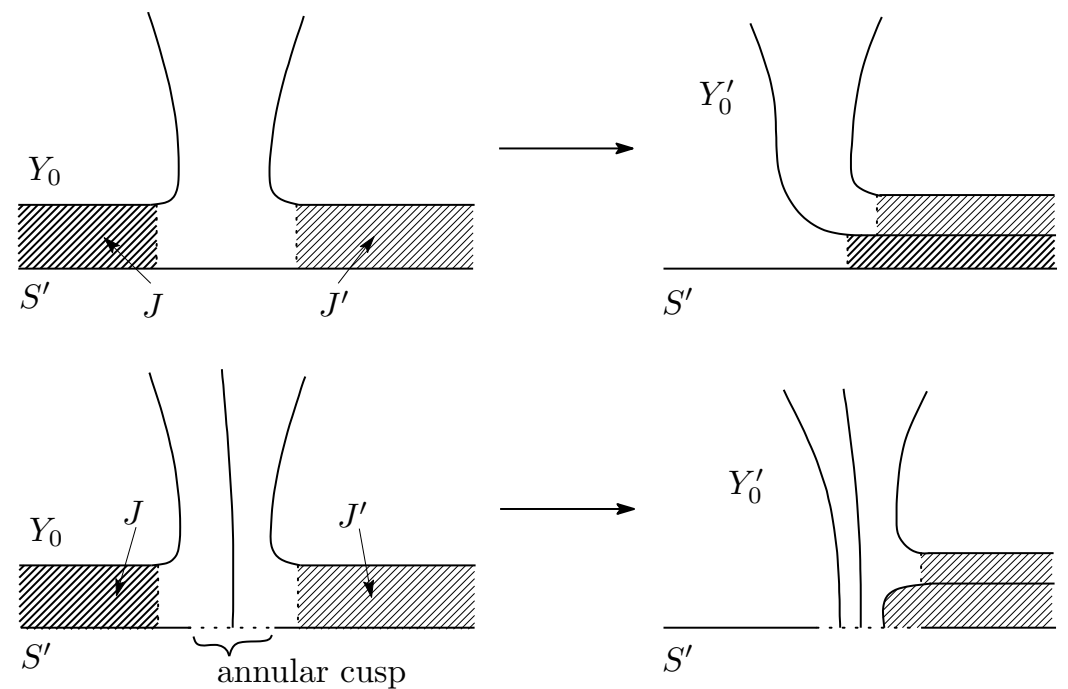

Figure 4. Constructions in the case where $S$ intersects 2 components of the window whose Euler characteristics are -1 .

lies on the same side as $p(J)$. Hence the above construction can be performed only finitely many times.

Let $Y_{1}$ be the essential surface obtained by performing the above construction as many times as possible. The Euler characteristic of the intersection of $S$ and the window of $L \rrbracket Y_{1}$ equals -1 . Therefore the Euler characteristic of the intersection of $S$ and $\operatorname{Guts}\left(L \rrbracket Y_{1}\right)$ is equal to -1 . In particular, $\operatorname{Guts}\left(L \rrbracket Y_{1}\right) \neq \varnothing$.

We will find an essential surface $Y_{2}$ such that $\chi\left(\partial \operatorname{Guts}\left(L \rrbracket Y_{2}\right)\right) \leq-4$. If $\chi\left(\partial \operatorname{Guts}\left(L \rrbracket Y_{1}\right)\right) \leq-4$, put $Y_{2}=Y_{1}$. Suppose that $\chi\left(\partial \operatorname{Guts}\left(L \rrbracket Y_{1}\right)\right)=-2$. Since the Euler characteristic of $\partial \operatorname{Guts}\left(L \rrbracket Y_{1}\right)-S^{\prime}$ is equal to -1 , it is either a 1-punctured torus or a 3-punctured sphere.

Suppose that $\partial \operatorname{Guts}\left(L \rrbracket Y_{1}\right)-S^{\prime}$ is a 1-punctured torus. Then $\partial \operatorname{Guts}\left(L \backslash Y_{1}\right)-S^{\prime}$ can contain a pared annulus, and $Y_{1} \cap \partial \operatorname{Guts}\left(L \rrbracket Y_{1}\right)$ is a 1-punctured torus or a 3-punctured sphere. If $Y_{1} \cap \partial \operatorname{Guts}\left(L \backslash Y_{1}\right)$ is a 1-punctured torus, let $Y_{1}^{\prime}$ be the surface which is the union of $Y_{1}-\left(Y_{1} \cap \partial \operatorname{Guts}\left(L \rrbracket Y_{1}\right)\right)$ and a surface in $p\left(J^{\prime}\right)$ (Figure 5). If $Y_{1} \cap \partial \operatorname{Guts}\left(L \rrbracket Y_{1}\right)$ is a 3-punctured sphere, we obtain the surface $\tilde{Y}_{1}$ by modifying $Y_{1}$ around the pared annulus in $\partial \operatorname{Guts}\left(L \rrbracket Y_{1}\right)-S^{\prime}$ (Figure 6). Here $\tilde{Y}_{1} \cap \partial \operatorname{Guts}\left(L \rrbracket Y_{1}\right)$ is a 1-punctured torus. Thus we obtain an essential surface $Y_{1}^{\prime}$ as the union of $\tilde{Y}_{1}-\left(\tilde{Y}_{1} \cap \partial \operatorname{Guts}\left(L \rrbracket Y_{1}\right)\right)$ and a surface in $p\left(J^{\prime}\right)$ (Figure 5).

Suppose that $\partial \operatorname{Guts}\left(L \rrbracket Y_{1}\right)-S^{\prime}$ is a 3-punctured sphere. $\partial \operatorname{Guts}\left(L \rrbracket Y_{1}\right)-S^{\prime}$ does not contain a pared annulus. Let $Y_{1}^{\prime}$ be the surface which is the union of $Y_{1}-\left(Y_{1} \cap \partial \operatorname{Guts}\left(L \rrbracket Y_{1}\right)\right)$ and a surface in $p\left(J^{\prime}\right)$. 

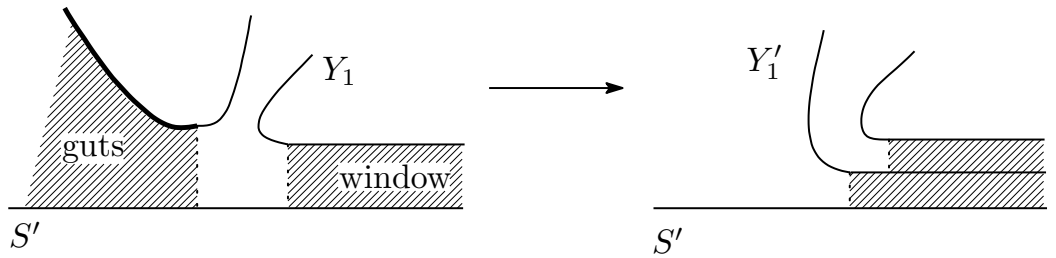

$S^{\prime}$
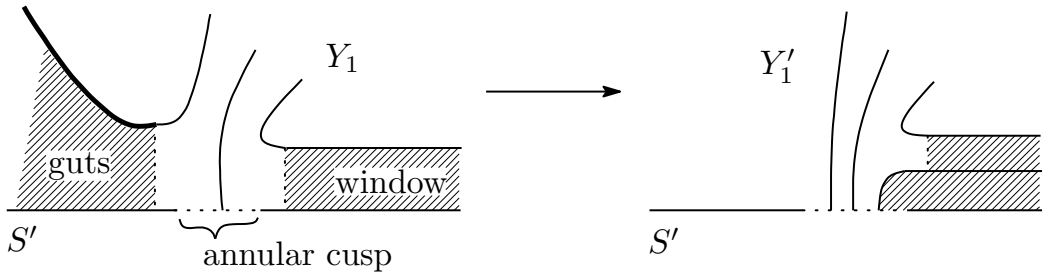

Figure 5. Constructions in the case where $\partial \operatorname{Guts}\left(L \rrbracket Y_{1}\right)=-2$.

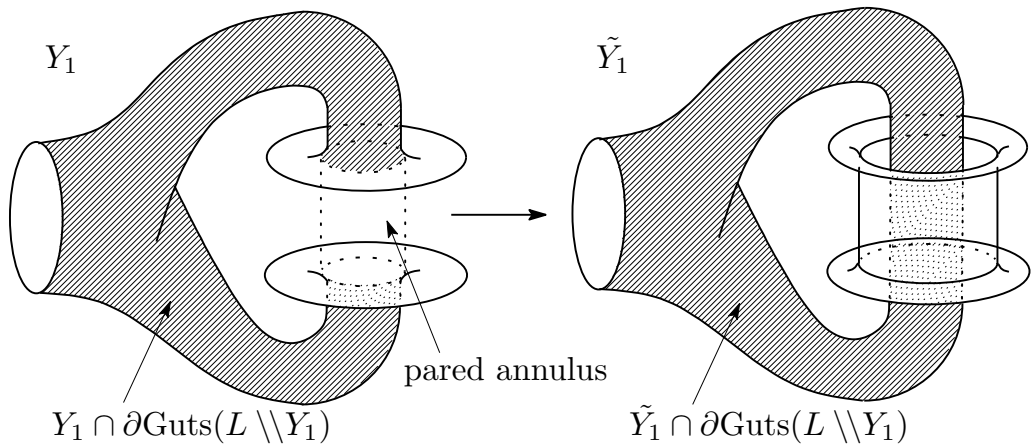

Figure 6. A construction around a pared annulus in the boundary of $\operatorname{Guts}\left(L \rrbracket Y_{1}\right)-S^{\prime}$.

We have obtained a surface $Y_{1}^{\prime}$ in these ways. Then, in general, we have $\left[Y_{1}^{\prime}, \partial Y_{1}^{\prime}\right] \neq\left[Y_{1}, \partial Y_{1}\right] \in H_{2}(L, \partial L ; \mathbb{Z})$, but $\left[\partial Y_{1}^{\prime}\right]=\left[\partial Y_{1}\right] \neq 0 \in H_{1}(\partial L ; \mathbb{Z})$. Since $\left|\chi\left(Y_{1}\right)\right|=\left|\chi\left(Y_{0}\right)\right|, Y_{1}$ is essential.

Since $Y_{1} \cap \partial \operatorname{Guts}\left(L \rrbracket Y_{1}\right)$ cannot be contained in the window of $L \rrbracket Y_{1}^{\prime}$, it follows that $S \cap \partial \operatorname{Guts}\left(L \rrbracket Y_{1}\right)$ is not contained in the window of $L \rrbracket Y_{1}^{\prime}$. Hence we can consider that $\operatorname{Guts}\left(L \rrbracket Y_{1}^{\prime}\right)$ contains $S \cap \partial \operatorname{Guts}\left(L \rrbracket Y_{1}\right)$. Since $Y_{1}$ is essential, the added surface in the window is not contained in $Y_{1}^{\prime} \cap \partial \operatorname{Guts}\left(L \rrbracket Y_{1}^{\prime}\right)$. Hence the above construction can be performed only finitely many times. 
Let $Y_{2}$ be the essential surface obtained by performing the above construction as many times as possible. Then $\chi\left(\partial \operatorname{Guts}\left(L \rrbracket Y_{2}\right)\right)$ is no longer equal to -2 , and $\chi\left(\partial \operatorname{Guts}\left(L \rrbracket Y_{2}\right)\right) \leq-4$.

(ii) Suppose that $S$ intersects no component of the window of $L \rrbracket Y_{0}$. Then $\chi\left(\operatorname{Guts}\left(L \rrbracket Y_{0}\right) \cap S\right)=-2$. Assume that $\chi\left(\partial \operatorname{Guts}\left(L \rrbracket Y_{0}\right)\right)=-2$. $\partial \operatorname{Guts}\left(L \rrbracket Y_{0}\right)$ is a closed surface which is the union of a surface in $S$ and annuli. Since $L$ is atoroidal, $\partial \operatorname{Guts}\left(L \rrbracket Y_{0}\right)$ contains the closed surface $S^{\prime}$. Hence $\partial \operatorname{Guts}\left(L \rrbracket Y_{0}\right)$ consists of $S^{\prime}$ and some torus cusps of $L$. The connectivity of $L$ implies that $L=\operatorname{Guts}\left(L \rrbracket Y_{0}\right)$. It contradicts that $Y_{0}$ is nonempty. Therefore $\chi\left(\partial \operatorname{Guts}\left(L \rrbracket Y_{0}\right)\right) \leq-4$.

We prove the essential part of Theorem 1.2.

Theorem 4.3. Let $M$ be an orientable hyperbolic manifold with 4 cusps. Then $\operatorname{vol} M \geq 2 V_{8}$. Moreover, if vol $M=2 V_{8}, M$ is obtained from two ideal regular octahedra by gluing along the faces.

Proof. It is sufficient to find an essential surface $X \subset M$ such that $\chi(\partial \operatorname{Guts}(X)) \leq$ -4 . Then Theorem 4.3 follows from Theorem 2.5.

Let $T_{1}, \ldots, T_{4}$ be the cusps of $M$. We take an essential surface $X_{0}$ such that $X_{0} \cap T_{1} \neq \varnothing$ and $X_{0} \cap T_{i}=\varnothing(2 \leq i \leq 4)$ by Theorem 2.11. We perform annular compressions for $X_{0}$ as many times as possible to obtain an essential surface $X_{1}$. When annular compression is performed, the number of boundary components of the surface increases and its Euler characteristic does not change. Since the Euler characteristic of each component of the obtained essential surface is negative, annular compressions can be performed only finitely many times.

We will show that Guts $\left(X_{1}\right)$ intersects $T_{2}, \ldots, T_{4}$. Let $k$ be the number of cusps intersecting $X_{1}(1 \leq k \leq 4)$. Let $T_{1}, \ldots, T_{k}$ be the cusps intersecting $X_{1}$. Let $A_{2}, \ldots, A_{k}$ be the annuli in $T_{2} \backslash \partial X_{1}, \ldots, T_{k} \backslash \partial X_{1}$ created by the last annular compressions to $T_{2}, \ldots, T_{k}$. Since there are no compressing annuli any more, Lemma 2.10 implies that $A_{2}, \ldots, A_{k}$ are not contained in a solid torus component of the JSJ decomposition of $M \| X_{1}$ and $T_{k+1}, \ldots, T_{4}$ are not contained in a $T^{2} \times I$ component of it. Since compressing annuli to different cusps can be taken disjointly, we may change the order of annular compressions to different cusps. By Lemma $2.9, A_{2}, \ldots, A_{k}$ are not contained in the window of $M \rrbracket X_{1}$. Therefore $A_{2}, \ldots, A_{k}, T_{k+1}, \ldots, T_{4} \subset \partial \operatorname{Guts}\left(X_{1}\right)$.

If $\operatorname{Guts}\left(X_{1}\right)$ is not connected, then $\chi\left(\partial \operatorname{Guts}\left(X_{1}\right)\right) \leq-4$ as desired. Suppose that $\operatorname{Guts}\left(X_{1}\right)$ is connected. Then $A_{2}, \ldots, A_{k}, T_{k+1}, \ldots, T_{4}$ are contained in one component $N$ of $M \backslash X_{1}$. We will find an essential surface $X_{2}$ such that $\partial \operatorname{Guts}\left(X_{2}\right)$ contains at least 4 pared components.

(i) Suppose that $\left(T_{1} \backslash \partial X_{1}\right) \cap N \neq \varnothing$. If $N=\operatorname{Guts}\left(X_{1}\right)$, let $A_{1}$ be an annulus which is a component of $\left(T_{1} \Downarrow \partial X_{1}\right) \cap N$. Otherwise let $A_{1}$ be an separating annulus of 
the JSJ decomposition intersecting $\operatorname{Guts}\left(X_{1}\right)$. In either case, $A_{1}$ is a pared annulus of Guts $\left(X_{1}\right)$ different from $A_{2}, \ldots, A_{k}$. Then it is sufficient to put $X_{2}=X_{1}$.

(ii) Suppose that $\left(T_{1} \backslash \partial X_{1}\right) \cap N=\varnothing$. Let $X_{1}^{\prime}=X_{1} \cap p(N)$, where $p: M \backslash X_{1} \rightarrow M$ is the natural projection. Then $X_{1}^{\prime}$ is an essential surface in $M$ and $T_{1} \cap X_{1}^{\prime}=\varnothing$. $X_{1}^{\prime}$ is the union of the components of $X_{1}$ intersecting $N$. If we cannot perform an annular compression for $X_{1}^{\prime}$ to $T_{1}$, Guts $\left(X_{1}^{\prime}\right)$ contains a neighborhood of $T_{1}$ which is in the complement of $N$. Since $\operatorname{Guts}\left(X_{1}^{\prime}\right)$ is not connected, $\chi\left(\partial \operatorname{Guts}\left(X_{1}^{\prime}\right)\right) \leq-4$. Then it is sufficient to put $X_{2}=X_{1}^{\prime}$.

If we can perform an annular compression for $X_{1}^{\prime}$ to $T_{1}$, we obtain $X_{2}$ by performing annular compressions to $T_{1}$ as many times as possible. Let $A_{1}$ be the innermost annulus in $T_{1}$. Since $X_{1}$ is obtained by performing annular compressions as many times as possible, there is no compressing annulus for $X_{1}^{\prime}$ to $A_{2}, \ldots, A_{k}, T_{k+1}, \ldots, T_{4}$ in $p(N)$. Hence there is no compressing annulus for $X_{2}$ to $A_{2}, \ldots, A_{k}, T_{k+1}, \ldots, T_{4}$ in $p(N)$. Since the surface which is obtained by filling $X_{1}^{\prime}$ with $A_{2}, \ldots, A_{k}$ consists of components of a surface in the process of the annular compression from $X_{0}$ to $X_{1}$, it is essential. This implies that $A_{1}, \ldots, A_{k}$ are not contained in the window of $M \backslash X_{2}$ by Lemma 2.9. Therefore $A_{1}, \ldots, A_{k}, T_{k+1}, \ldots, T_{4} \subset \operatorname{Guts}\left(X_{2}\right)$.

Finally, we will find an essential surface $X$ such that $\chi(\partial \operatorname{Guts}(X)) \leq-4$. If $k=4$, the 4 annuli $A_{1}, \ldots, A_{4}$ are disjoint and not homotopic to each other in the nontorus components of $\partial \operatorname{Guts}\left(X_{2}\right)$. This implies that $\chi\left(\partial \operatorname{Guts}\left(X_{2}\right)\right) \leq-4$. Then it is sufficient to put $X=X_{2}$.

If $1 \leq k \leq 3, \operatorname{vol} \operatorname{Guts}\left(X_{2}\right) \geq 2 V_{8}$ by Theorem 4.1. Therefore vol $M \geq 2 V_{8}$ by Theorem 2.5. But we need to find $X$ in order to prove that $M$ is obtained from 2 octahedra when the equality holds. Lemma 3.2 and Theorem 4.1 imply that there is an essential surface $Y$ in $\operatorname{Guts}\left(X_{2}\right)$ such that $\chi\left(\partial \operatorname{Guts}\left(\operatorname{Guts}\left(X_{2}\right) \rrbracket Y\right)\right) \leq-4$. Then $Y$ intersects some of $A_{1}, \ldots, A_{k}, T_{k+1}, \ldots, T_{4}$, where $A_{2}, \ldots, A_{k}, T_{k+1}, \ldots, T_{4}$ are contained in $\partial M$. If $A_{1}$ is contained in $\partial M$ or does not intersect $Y, X_{3} \cup Y$ is properly embedded in $M$. Since $\operatorname{Guts}\left(X_{2} \cup Y\right)=\operatorname{Guts}\left(\operatorname{Guts}\left(X_{2}\right) \Downarrow Y\right)$, we obtain $\chi\left(\partial \operatorname{Guts}\left(X_{2} \cup Y\right)\right) \leq-4$. Then it is sufficient to let $X=X_{2} \cup Y$. If $A_{1}$ is contained in the interior of $M$ and intersects $Y, X_{3} \cup Y$ is not properly embedded in $M$. Suppose that $A_{1} \cap Y$ is the union of $l$ simple closed curves. Let $X$ be the union of 2 surfaces parallel to $Y, X_{2} \cap \partial \operatorname{Guts}\left(X_{2}\right)$ and $l+1$ times of $X_{2}-\partial \operatorname{Guts}\left(X_{2}\right)$ (Figure 7). Since $\operatorname{Guts}(X)$ is homeomorphic to $\operatorname{Guts}\left(\operatorname{Guts}\left(X_{2}\right) \rrbracket Y\right), \chi(\partial \operatorname{Guts}(X)) \leq-4$.

\section{Realization of hyperbolic manifold}

In this section we will prove that an orientable hyperbolic 3-manifold obtained from 2 ideal regular octahedra by gluing along the faces is homeomorphic to the complement of the $8_{2}^{4}$ link. This completes the proof of Theorem 1.2. 

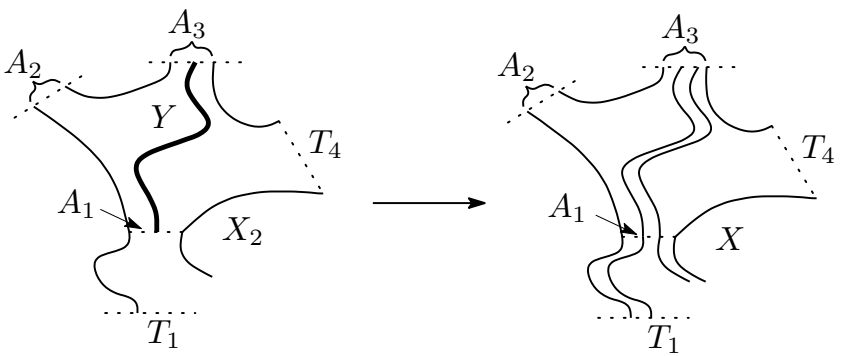

Figure 7. A construction of an essential surface the boundary of whose guts is no more than -4 .

Thurston [1980, Chapter 6, Example 6.8.6] calculated the volume of the complement of the $8_{2}^{4}$ link to be $2 V_{8}$. SnapPy [Culler and Dunfield unskip] has the list of the orientable hyperbolic 3-manifolds obtained from 8 ideal regular tetrahedra by gluing along the faces. These imply the uniqueness of the minimal volume orientable hyperbolic 3-manifold with 4 cusps, but we prove it here by an elementary argument examining the possible ways of gluing along the faces of 2 octahedra.

The 12 vertices of the 2 octahedra correspond to the 4 cusps of the hyperbolic manifold. We look at the number of vertices corresponding to each cusp. Since the edge angles of an ideal regular octahedron are right angles, 4 edges of the 2 octahedra should be glued together.

Claim 5.1. If there is a cusp consisting of one vertex $x$, the faces around $x$ are glued as in Figure 8(a). If there is a cusp consisting of 2 vertices $a$ and $b$, the faces around $a$ and $b$ are glued as in Figure 8(b).

Proof. If there is a cusp consisting of one vertex $x$, the 4 edges around $x$ are glued together, and each face around $x$ is glued with the opposite face.

Suppose there is a cusp consisting of 2 vertices $a$ and $b$. Assume that $a$ and $b$ are contained in one octahedron. If $a$ and $b$ are adjacent, no edges can be glued

(a)

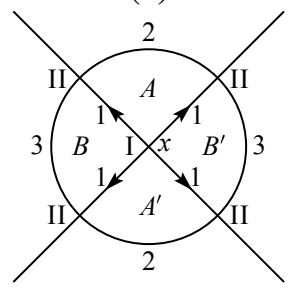

(b)

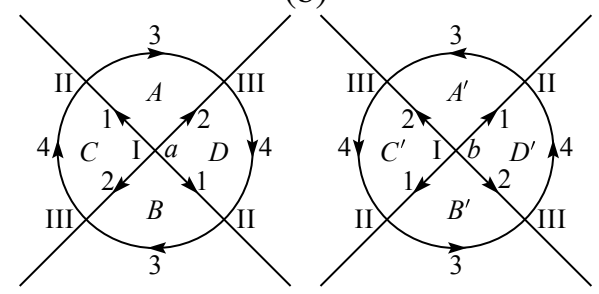

Figure 8. Face gluings for a one-vertex cusp (a) and a two-vertex cusp (b). In each case, edges with the same number are to be identified, and likewise with vertices. Unprimed faces are to be identified with their primed counterparts. 

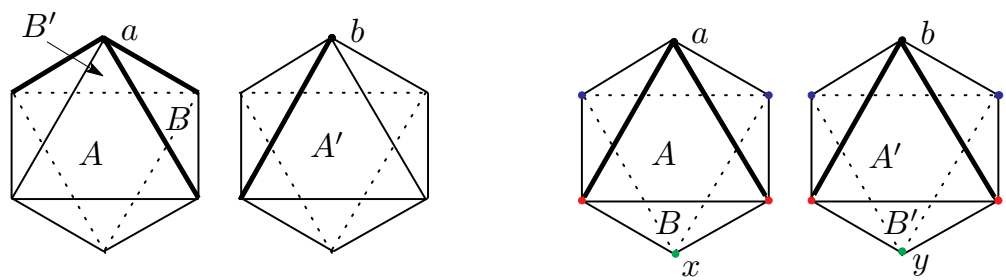

Figure 9. An impossible example in the case where two vertices form a cusp. Face $A$ is glued to $A^{\prime}$ and $B$ to $B^{\prime}$.

with the edge between $a$ and $b$. If $b$ is opposite to $a$, we can glue no pairs of faces which are contained in different octahedra. This contradicts the connectivity. Hence $a$ and $b$ are contained in different octahedra.

We consider how the 8 edges around $a$ are $b$ are glued. Since $a$ and $b$ are glued, the 4 edges around $a$ cannot be glued together. If 3 edges around $a$ and one edge around $b$ are glued together, 2 adjacent faces around $a$ are glued (the left of Figure 9). Then the edge between the 2 faces can be glued with no edges. Hence 2 edges around $a$ and 2 edges around $b$ are glued together. Assume that adjacent edges around $a$ are glued. Let $x$ and $y$ be the vertices opposite to $a$ and $b$, respectively. If $x$ and $y$ form 2 cusps with themselves, there are 2 edges glued with no other edges. Since there are 4 cusps, there is a cusp consisting of $x$ and $y$. There are 2 edges glued with no other edges even in this case (the right of Figure 9). Therefore opposite edges around $a$ are glued and the way of gluing is determined.

Claim 5.2. There is no cusp consisting of 3 vertices.

Proof. Assume that there is a cusp consisting of 3 vertices $a, b$ and $c$. If $a, b$ and $c$ are vertices of one octahedron, 2 positions are possible (the left of Figure 10). If $a, b$ and $c$ are the vertices of one face, this face cannot be glued with another face. Otherwise, at least one of $a, b$ and $c$ is contained in a face of the octahedron containing $a, b$ and $c$. This implies that no pair of faces of different octahedra can be glued. Hence $a, b$ and $c$ are not contained in one octahedron. We assume that $b$ and $c$ are contained in one octahedron without loss of generality. Then 2 positions are possible (the right of Figure 10). If $b$ and $c$ are adjacent, no edges can be glued with the edge between $b$ and $c$. Hence $c$ is opposite to $b$. Let $x$ be the vertex opposite to $a$. Since only the 4 faces around $x$ do not contain $a, b$ or $c$, the 4 faces cannot be glued with any faces of the other octahedron.

Assume that $x$ does not form a cusp with itself. Suppose that adjacent faces around $x$ are glued. Then the 5 vertices except $a$ of the octahedron containing $a$ are glued together. There are 2 vertices $y$ and $z$ which form 2 cusps with themselves on the octahedron containing $b$ and $c$. The 4 vertices around $y$ are glued together 

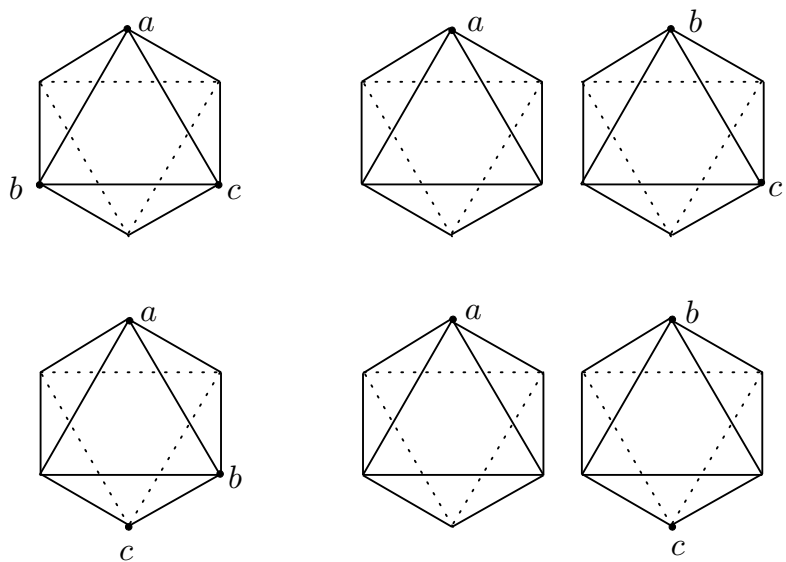

Figure 10. Positions of three vertices.

These points form two cusps with themselves.
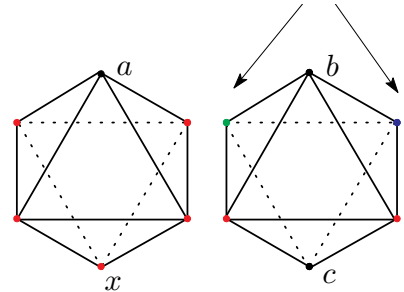

Figure 11. Five vertices of an octahedron cannot be glued together.

by Claim 5.1 (Figure 11). This contradicts that $b$ is glued only with $a$ and $c$. Hence opposite faces around $x$ are glued.

Suppose that opposite faces $A$ and $B$ around $x$ are glued with a twist, that is, the 2 vertices corresponding with $x$ in $A$ and $B$ are not glued. Then 2 opposite vertices on the octahedron containing $a$ are glued with $x$. Since the 5 vertices except $a$ of the octahedron containing $a$ cannot be glued together, the other faces $C$ and $D$ around $x$ are glued with a twist. The 4 faces around $a$ are glued with faces of the other octahedron because of the correspondence of the vertices and the fact that adjacent faces around $a$ cannot be glued. Hence there is a vertex which forms a cusp with itself on the octahedron containing $b$ and $c$ (Figure 12). This contradicts that $b$ is glued only with $a$ and $c$.

Hence $x$ forms a cusp with itself. Since 4 edges are glued together, the faces around $a$ are glued with faces of the other octahedron. At least 3 vertices of the octahedron containing $b$ and $c$ are glued with the 4 vertices except $a$ and $x$. Since we must obtain 4 cusps, there is a vertex that forms a cusp with itself. This is a contradiction. 
This point forms a cusp with itself.
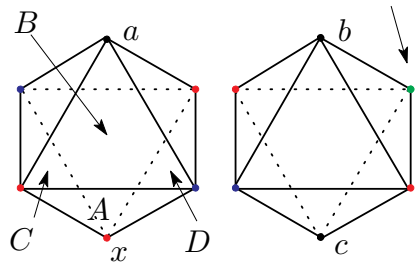

Figure 12. Opposite faces cannot be glued with a twist.
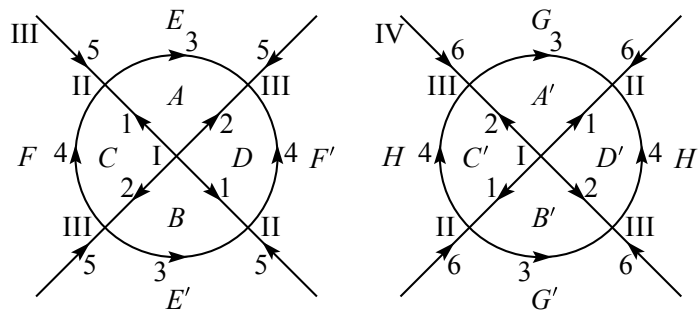

Figure 13. First gluing of the octahedra.

Claim 5.2 implies that there is a cusp consisting of one or 2 vertices. Suppose that there is a cusp consisting of one vertex $x$. The 4 vertices around $x$ are glued together. The 4 faces $A, B, C$ and $D$ around the vertex $a$ opposite to $x$ are glued with faces of the other octahedron. Vertex $a$ is glued with only one vertex $b$ because of Claim 5.2 and the fact that 7 vertices are glued. Since the 8 vertices around $a$ and $b$ are glued together, the vertex $y$ opposite to $b$ forms a cusp with itself. The numbers of the vertices corresponding to the cusps are 1, 1,2 and 8. By Claim 5.1 the way of gluing is determined as in Figure 13.

Suppose that there is no cusp consisting of one vertex. Then there is a cusp consisting of 2 vertices $a$ and $b . A, A^{\prime}, B, B^{\prime}, C, C^{\prime}, D$ and $D^{\prime}$ around $a$ and $b$ are glued as Figure 8. Since no cusp consists of one vertex, the 2 vertices $x$ and $y$ opposite to $a$ and $b$, respectively, are glued together. The numbers of the vertices corresponding to the cusps are 2, 2, 4 and 4. The face $E$ adjacent to $A$ is glued with the face $E^{\prime}$ adjacent to $B^{\prime}$ because of the correspondence of the vertices and edges. The way of gluing is determined as in Figure 14.
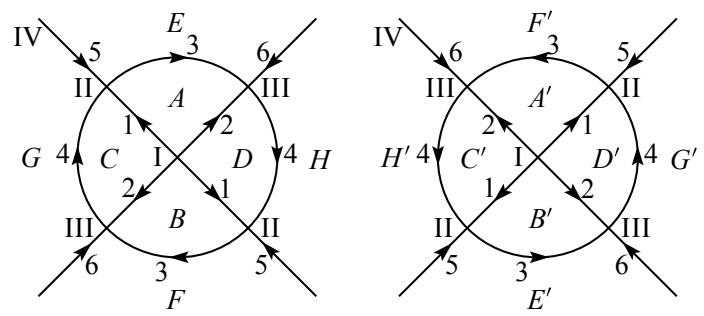

Figure 14. Second gluing of the octahedra. 
Both gluings of the octahedra give homeomorphic spaces by Figure 15 and they are the $8_{2}^{4}$ link complements by Figure 16 .
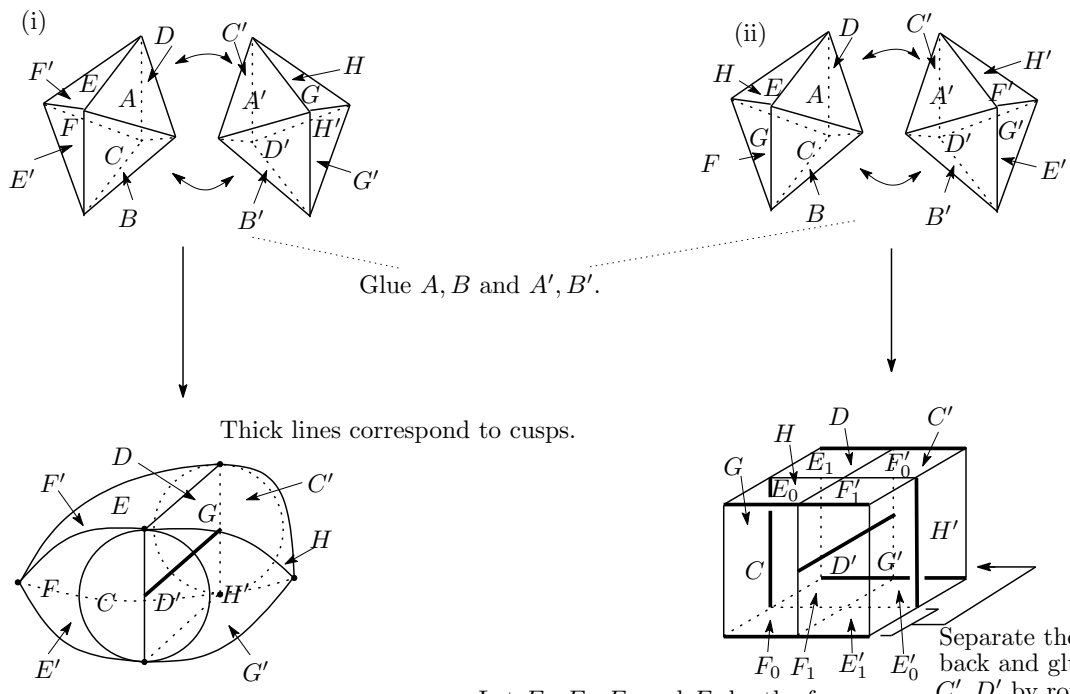

Glue $A, B$ and $A^{\prime}, B^{\prime}$.

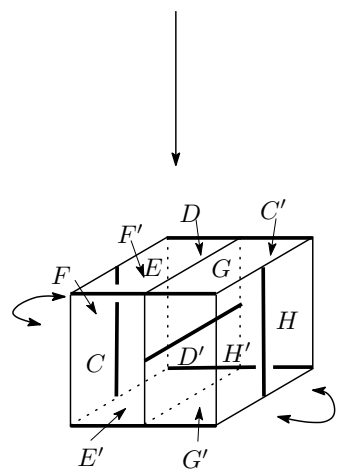

Let $E_{0}, E_{1}, F_{0}$ and $F_{1}$ be the faces

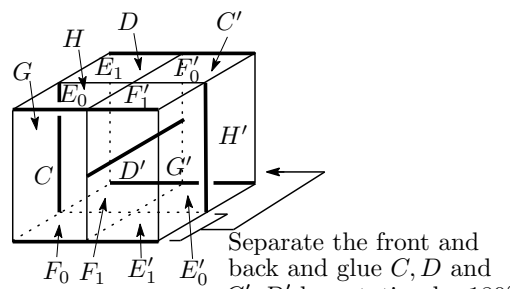
obtained by dividing $E$ and $F$. $C^{\prime}, D^{\prime}$ by rotating by $180^{\circ}$.

Rotate by $90^{\circ}$ around the central thick line.

The ways of gluing are identical though the ways of division are different.

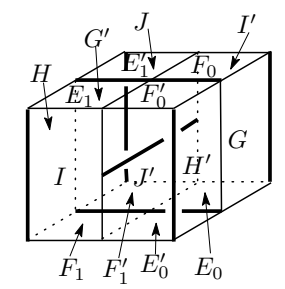

Let $I, I^{\prime}, J$ and $J^{\prime}$ be the new sections.

Glue $F, H$ and $F^{\prime}, H^{\prime}$.

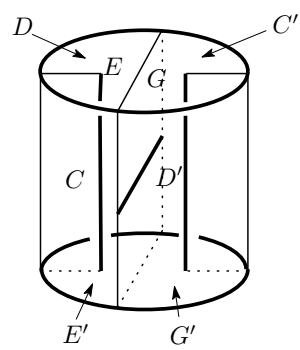

Figure 15. The gluings from Figures 13 and 14 (replicated in diagrams (i) and (ii) at the top) lead to homeomorphic spaces. 


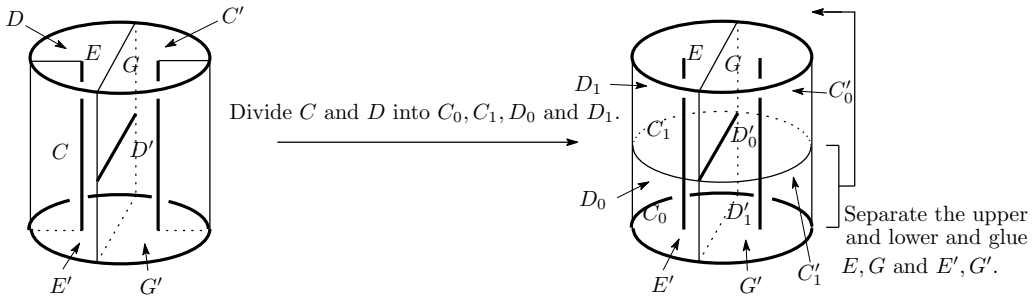

We can twist them along the thick loops.

Rotate the right half by $180^{\circ}:$

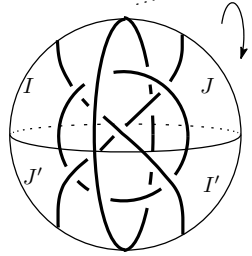

Glue $C_{i}, D_{i}$ and $C_{i}^{\prime}, D_{i}^{\prime}$.

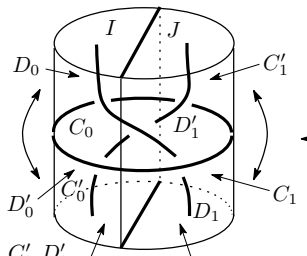

$J^{\prime}$

Glue $I, J$ and $I^{\prime}, J^{\prime}$.
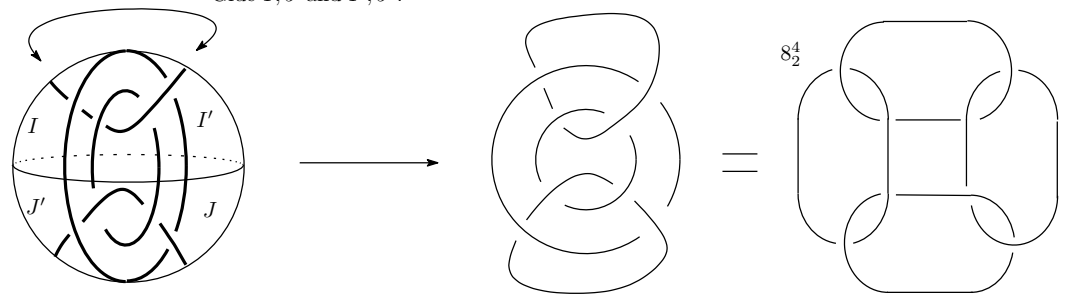

Figure 16. The space obtained in Figure 15 is the complement of the $8_{2}^{4}$ link.

\section{Acknowledgement}

The author would like to express his gratitude to Takashi Tsuboi for helpful guidance and advice.

\section{References}

[Adams 1988] C. C. Adams, "Volumes of $N$-cusped hyperbolic 3-manifolds", J. London Math. Soc. (2) 38:3 (1988), 555-565. MR 89k:22020 Zbl 0627.57013

[Agol 2010] I. Agol, "The minimal volume orientable hyperbolic 2-cusped 3-manifolds", Proc. Amer. Math. Soc. 138:10 (2010), 3723-3732. MR 2011k:57023 Zbl 1203.57006 
[Agol et al. 2007] I. Agol, P. A. Storm, and W. P. Thurston, "Lower bounds on volumes of hyperbolic Haken 3-manifolds", J. Amer. Math. Soc. 20:4 (2007), 1053-1077. MR 2008i:53086 Zbl 1155.58016

[Benedetti and Petronio 1992] R. Benedetti and C. Petronio, Lectures on hyperbolic geometry, Springer, Berlin, 1992. MR 94e:57015 Zbl 0768.51018

[Calegari et al. 2010] D. Calegari, M. H. Freedman, and K. Walker, "Positivity of the universal pairing in 3 dimensions", J. Amer. Math. Soc. 23:1 (2010), 107-188. MR 2011k:57037 Zbl 1201.57024

[Cao and Meyerhoff 2001] C. Cao and G. R. Meyerhoff, "The orientable cusped hyperbolic 3manifolds of minimum volume", Invent. Math. 146:3 (2001), 451-478. MR 2002i:57016 Zbl 1028. 57010

[Culler and Dunfield unskip] M. Culler and N. Dunfield, "SnapPy: a computer program for studying the geometry and topology of 3-manifolds", http://snappy.computop.org.

[Culler and Shalen 1984] M. Culler and P. B. Shalen, "Bounded, separating, incompressible surfaces in knot manifolds", Invent. Math. 75:3 (1984), 537-545. MR 85k:57010 Zbl 0542.57011

[Gabai et al. 2009] D. Gabai, R. Meyerhoff, and P. Milley, "Minimum volume cusped hyperbolic three-manifolds", J. Amer. Math. Soc. 22:4 (2009), 1157-1215. MR 2011a:57031 Zbl 1204.57013

[Hatcher 2007] A. Hatcher, "Notes on basic 3-manifold topology", 2007, available at http://www.math. cornell.edu/ hatcher/3M/3Mdownloads.html

[Jaco and Shalen 1979] W. H. Jaco and P. B. Shalen, "Seifert fibered spaces in 3-manifolds", Mem. Amer. Math. Soc. 21:220 (1979), viii+192. MR 81c:57010 Zbl 0415.57005

[Johannson 1979] K. Johannson, Homotopy equivalences of 3-manifolds with boundaries, Lecture Notes in Mathematics 761, Springer, Berlin, 1979. MR 82c:57005 Zbl 0412.57007

[Leininger 2006] C. J. Leininger, "Small curvature surfaces in hyperbolic 3-manifolds", J. Knot Theory Ramifications 15:3 (2006), 379-411. MR 2007a:57025 Zbl 1090.57012

[Miyamoto 1994] Y. Miyamoto, "Volumes of hyperbolic manifolds with geodesic boundary", Topology 33:4 (1994), 613-629. MR 95h:57014 Zbl 0824.53038

[Morgan 1984] J. W. Morgan, "On Thurston's uniformization theorem for three-dimensional manifolds", pp. 37-125 in The Smith conjecture (New York, 1979), edited by J. W. Morgan and B. Hyman, Pure Appl. Math. 112, Academic Press, Orlando, FL, 1984. MR 758464 Zbl 0599.57002

[Ratcliffe 2006] J. G. Ratcliffe, Foundations of hyperbolic manifolds, 2nd ed., Graduate Texts in Mathematics 149, Springer, New York, 2006. MR 2007d:57029 Zbl 1106.51009

[Thurston 1980] W. P. Thurston, "The geometry and topology of 3-manifolds", lecture notes, 1980, http://library.msri.org/books/gt3m.

[Thurston 1986] W. P. Thurston, "Hyperbolic structures on 3-manifolds, I: Deformation of acylindrical manifolds”, Ann. of Math. (2) 124:2 (1986), 203-246. MR 88g:57014 Zbl 0668.57015

Received September 7, 2012. Revised January 24, 2013.

\section{KEN'ICHI YOSHIDA}

GRAduATE SCHOOL of MATHEMATICAL SCIENCES

UNIVERSITY OF TOKYO

TOKYO 153-8914

JAPAN

kyoshida@ms.u-tokyo.ac.jp 


\title{
PACIFIC JOURNAL OF MATHEMATICS
}

\author{
msp.org/pjm
}

Founded in 1951 by E. F. Beckenbach (1906-1982) and F. Wolf (1904-1989)

\section{EDITORS}

V. S. Varadarajan (Managing Editor)

Department of Mathematics

University of California

Los Angeles, CA 90095-1555

pacific@math.ucla.edu

Paul Balmer

Department of Mathematics

University of California

Los Angeles, CA 90095-1555

balmer@math.ucla.edu

Daryl Cooper

Department of Mathematics

University of California

Santa Barbara, CA 93106-3080 cooper@math.ucsb.edu

Jiang-Hua Lu

Department of Mathematics

The University of Hong Kong

Pokfulam Rd., Hong Kong jhlu@maths.hku.hk
Don Blasius

Department of Mathematics University of California

Los Angeles, CA 90095-1555

blasius@math.ucla.edu

Robert Finn

Department of Mathematics Stanford University

Stanford, CA 94305-2125

finn@math.stanford.edu

Sorin Popa

Department of Mathematics

University of California

Los Angeles, CA 90095-1555 popa@math.ucla.edu

Paul Yang

Department of Mathematics Princeton University

Princeton NJ 08544-1000

yang@math.princeton.edu

\section{PRODUCTION}

Silvio Levy, Scientific Editor, production@msp.org

\section{SUPPORTING INSTITUTIONS}

ACADEMIA SINICA, TAIPEI

CALIFORNIA INST. OF TECHNOLOGY

INST. DE MATEMÁTICA PURA E APLICADA

KEIO UNIVERSITY

MATH. SCIENCES RESEARCH INSTITUTE

NEW MEXICO STATE UNIV.

OREGON STATE UNIV.

\author{
STANFORD UNIVERSITY \\ UNIV. OF BRITISH COLUMBIA \\ UNIV. OF CALIFORNIA, BERKELEY \\ UNIV. OF CALIFORNIA, DAVIS \\ UNIV. OF CALIFORNIA, LOS ANGELES \\ UNIV. OF CALIFORNIA, RIVERSIDE \\ UNIV. OF CALIFORNIA, SAN DIEGO \\ UNIV. OF CALIF., SANTA BARBARA
}

\author{
Vyjayanthi Chari \\ Department of Mathematics \\ University of California \\ Riverside, CA 92521-0135 \\ chari@math.ucr.edu \\ Kefeng Liu \\ Department of Mathematics \\ University of California \\ Los Angeles, CA 90095-1555 \\ liu@math.ucla.edu \\ Jie Qing \\ Department of Mathematics \\ University of California \\ Santa Cruz, CA 95064 \\ qing@cats.ucsc.edu
}

These supporting institutions contribute to the cost of publication of this Journal, but they are not owners or publishers and have no responsibility for its contents or policies.

See inside back cover or msp.org/pjm for submission instructions.

The subscription price for 2013 is US \$400/year for the electronic version, and \$485/year for print and electronic.

Subscriptions, requests for back issues and changes of subscribers address should be sent to Pacific Journal of Mathematics, P.O. Box 4163, Berkeley, CA 94704-0163, U.S.A. The Pacific Journal of Mathematics is indexed by Mathematical Reviews, Zentralblatt MATH, PASCAL CNRS Index, Referativnyi Zhurnal, Current Mathematical Publications and Web of Knowledge (Science Citation Index).

The Pacific Journal of Mathematics (ISSN 0030-8730) at the University of California, c/o Department of Mathematics, 798 Evans Hall \#3840, Berkeley, CA 94720-3840, is published twelve times a year. Periodical rate postage paid at Berkeley, CA 94704, and additional mailing offices. POSTMASTER: send address changes to Pacific Journal of Mathematics, P.O. Box 4163, Berkeley, CA 94704-0163.

PJM peer review and production are managed by EditFLOW ${ }^{\circledR}$ from Mathematical Sciences Publishers.

\section{PUBLISHED BY}

mathematical sciences publishers

nonprofit scientific publishing

http://msp.org/

(C) 2013 Mathematical Sciences Publishers 


\section{PACIFIC JOURNAL OF MATHEMATICS}

Volume $266 \quad$ No. $2 \quad$ December 2013

Rate of attraction for a semilinear wave equation with variable coefficients and

critical nonlinearities

FÁGNER DiAs ARARUNA and FLANK DAVID MORAIS BEZERRA

The Brin-Thompson groups $s V$ are of type $\mathrm{F}_{\infty}$

Martin G. Fluch, Marco Marschler, Stefan Witzel and

MATTHEW C. B. ZAREMSKY

Ideal decompositions of a ternary ring of operators with predual

MASAYOSHI KANEDA

A study of real hypersurfaces with Ricci operators in 2-dimensional complex space 305 forms

Dong Ho Lim, WoOn Ha SoHn and Hyunjung Song

On commensurability of fibrations on a hyperbolic 3-manifold

HideTOSHI MASAI

Multiplicative Dirac structures

CRISTIÁN ORTIZ

On the finite generation of a family of Ext modules

TONY J. PuthenPuRAKAL

Index formulae for Stark units and their solutions

XAVIER-FranÇOIS ROBLOT

The short time asymptotics of Nash entropy

GUOYI XU

Several splitting criteria for vector bundles and reflexive sheaves

STEPHEN S.-T. YAU and FEI YE

The minimal volume orientable hyperbolic 3-manifold with 4 cusps

KEN'ICHI YOSHIDA

On the Witten rigidity theorem for string ${ }^{c}$ manifolds

JIANQING YU and BO LIU 